\title{
Research Article \\ Vibration Analysis of an Optical Fiber Coupler Using the Differential Quadrature Method
}

\author{
Ming-Hung Hsu \\ Department of Electrical Engineering, National Penghu University, Penghu, Taiwan \\ Correspondence should be addressed to Ming-Hung Hsu, hsu.mh@msa.hinet.net
}

Received 30 May 2009; Revised 30 October 2009; Accepted 18 February 2010

Academic Editor: David Chelidze

Copyright (C) 2010 Ming-Hung Hsu. This is an open access article distributed under the Creative Commons Attribution License, which permits unrestricted use, distribution, and reproduction in any medium, provided the original work is properly cited.

This study elucidates the dynamic characteristics of an optical fiber coupler via the differential quadrature simulations. A novel modeling scheme is suitable for developing an optical fiber coupler application. Exactly how the locations of bonding points, string tension, and spring stiffness of the rubber pad affect the dynamic behavior of the optical fiber coupler is investigated.

\section{Introduction}

An optical fiber coupler is an optical device with several input fibers and several output fibers. The optical fibers in couplers may be under shock and impact. Cheng and $\mathrm{Zu}$ [1] and Sun [2] studied vibration of an optical fiber coupler subjected to a half-sine shock. Malomed and Tasgal [3] analyzed the dynamics of small internal vibrations in a two-component gap soliton. They found three oscillation modes, which are composed of dilation-contraction of each component's width, and a relative translation of the two components. Brown et al. [4] performed vibration tests on commercial grade fiber optic connectors and splices. Huang et al. [5] presented optical coupling loss and vibration characterization for packaging of $2 \times 2$ MEMS vertical torsion mirror switches. Thomes et al. [6] presented vibration performance of current fiber optic connector.

In this study the idea of differential quadrature formulation is extended to an optical fiber coupler. During the last decade, the differential quadrature approach applied to engineering and science problems has attracted considerable attention [7-31]. Liew et al. [716] applied the differential quadrature method to Mindlin plates on Winkler foundations and developed an application of the differential quadrature method to thick symmetric cross-ply laminates with first-order shear flexibility. Liew et al. [7-16] also employed the generalized differential quadrature method for buckling analysis and examined static and free vibration 
of beams and rectangular and annular plates. Sherbourne and Pandey [17] investigated the buckling behavior of beams and composite plates using the differential quadrature method. Mirfakhraei and Redekop [18] evaluated the buckling of circular cylindrical shells using the differential quadrature method. Tomasiello [19] applied the differential quadrature method to evaluate initial-boundary-value problems. Moradi and Taheri [20] conducted buckling analysis of general laminated composite beams using the differential quadrature method. De Rosa and Franciosi [21] solved the dynamic problem of circular arches using the differential quadrature method. Sun and Zhu [22] investigated incompressible viscous flow problems using the differential quadrature method. Via the differential quadrature method, Tanaka and Chen [23] solved transient elastodynamic problems. Chen and Zhong [24] observed that the differential quadrature and differential cubature methods, due to their global domain characteristics, are more efficient in solving nonlinear problems than conventional numerical schemes, such as the finite element and finite difference methods. Civan [25] solved multivariable mathematical models using the differential quadrature and differential cubature methods. Hua and Lam [26] identified the frequency characteristics of a thin rotating cylindrical shell using the differential quadrature method. Wang et al. [27-30] employed new versions of the differential quadrature and differential quadrature element methods to analyze anisotropic rectangular plates, frame structures, nonuniform beams, circular annular plates, and isotropic skew plates. The dynamic behavior of an optical fiber coupler is elucidated using the differential quadrature method in this work. Few studies have conducted vibration analysis of an optical fiber coupler using the differential quadrature method.

\section{Differential Quadrature Method}

Solutions to numerous complex beam problems have been efficiently acquired using fast computers and various numerical schemes, including the Galerkin technique, finite element method, boundary element method, and Rayleigh-Ritz method. In this study, the differential quadrature scheme is employed to generate discrete eigenvalue problems for an optical fiber coupler. The basic concept of the differential quadrature method is that the derivative of a function at a given point can be approximated as a weighted linear sum of functional values at all sample points in the domain of that variable. The partial differential equation is then reduced to a set of algebraic equations. For a function, $f(x)$, the differential quadrature approximation for the $m$ th-order derivative at the $i$ th sample point is given by

$$
\left[\begin{array}{c}
\frac{d^{m} f\left(x_{1}\right)}{d x^{m}} \\
\frac{d^{m} f\left(x_{2}\right)}{d x^{m}} \\
\vdots \\
\frac{d^{m} f\left(x_{N}\right)}{d x^{m}}
\end{array}\right] \cong\left[D_{i j}^{(m)}\right]\left[\begin{array}{c}
f\left(x_{1}\right) \\
f\left(x_{2}\right) \\
\vdots \\
f\left(x_{N}\right)
\end{array}\right] \text { for } i, j=1,2, \ldots, N
$$

where $f\left(x_{i}\right)$ is the value of the function at sample point $x_{i}, D_{i j}^{(m)}$ is the weighted coefficient of the $m$ th-order differentiation attached to these functional values, $N$ is the number of sample points, and $x_{i}$ is the location of the $i$ th sample point in the domain. The most convenient 
technique is to distribute sample points uniformly [31]. A Lagrangian interpolation polynomial is utilized to eliminate possible adverse conditions when determining the weighted coefficients $D_{i j}^{(m)}$ [31], which are as follows:

$$
f(x)=\frac{M(x)}{\left(x-x_{i}\right) M_{1}\left(x_{i}\right)} \quad \text { for } i=1,2, \ldots, N,
$$

where

$$
\begin{aligned}
M(x) & =\prod_{j=1}^{N}\left(x-x_{j}\right), \\
M_{1}\left(x_{i}\right) & =\prod_{j=1, j \neq i}^{N}\left(x_{i}-x_{j}\right) \quad \text { for } i=1,2, \ldots, N .
\end{aligned}
$$

Inputting (2.2) into (2.1) yields

$$
\begin{aligned}
& D_{i j}^{(1)}=\frac{M_{1}\left(x_{i}\right)}{\left(x_{i}-x_{j}\right) M_{1}\left(x_{j}\right)} \quad \text { for } i, j=1,2, \ldots, N, i \neq j, \\
& D_{i i}^{(1)}=-\sum_{j=1, j \neq i}^{N} D_{i j}^{(1)} \text { for } i=1,2, \ldots, N .
\end{aligned}
$$

The coefficients of the weighted matrix can be acquired using (2.4). For the mth-order derivative, the weighted coefficients can be obtained using the following recurrence relation equations:

$$
D_{i j}^{(m)}=\sum_{k=1}^{N} D_{i k}^{(1)} D_{k j}^{(m-1)} \quad \text { for } i, j=1,2, \ldots, N
$$

The selection of sample points always is very important to solution accuracy when using the differential quadrature approach. For a beam problem, the most convenient technique is to choose equally spaced sample points [31]. Unequally spaced sample points, such as Chebyshev-Gauss-Lobatto sample points, have been utilized by a number of studies. With the Chebyshev-Gauss-Lobatto distribution, the sample points of an optical fiber coupler are distributed as

$$
x_{i}=\frac{1}{2}\left(1-\cos \frac{(i-1) \pi}{N-1}\right) \text { for } i=1,2, \ldots, N
$$

\section{Vibration of an Optical Fiber Coupler with a Continuous Elastic Support}

Figure 1 shows a sectional view of the optical fiber coupler with a continuous elastic support. The fibers, the substrate, and the rubber pad are on the inside of the steel tube. The optical 


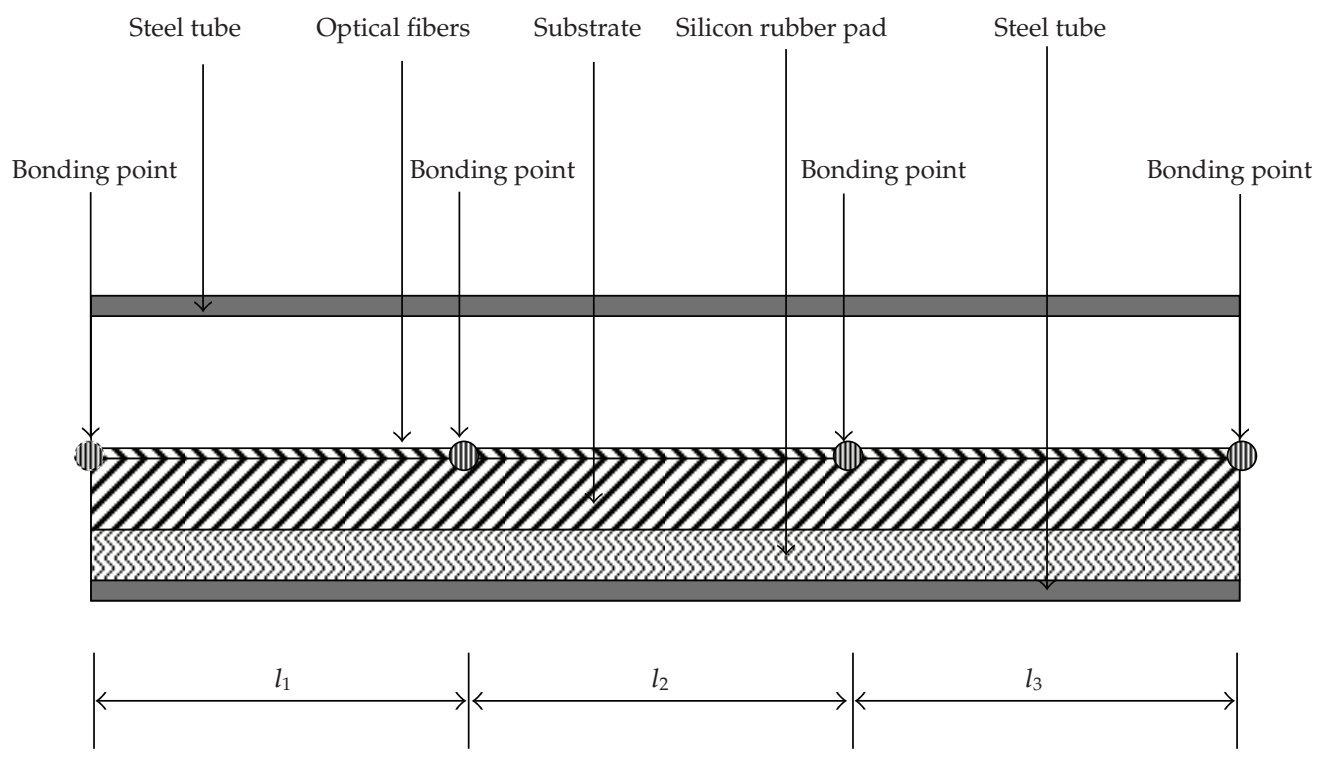

Figure 1: Sectional view of the optical fiber coupler with an elastic foundation $[1,2]$.

fibers are placed onto the substrate. A beam represents the substrate. The optical fibers model as a string. The rubber pad is placed between the substrate and steel tube. The linear model assumes that fiber tension is constant. The equation of motion for the optical fiber coupler is derived as $[1,2]$

$$
\begin{gathered}
P \frac{\partial^{2} u_{1}}{\partial x^{2}}-\rho_{1} A_{1} \frac{\partial^{2} u_{1}}{\partial t^{2}}=0 \quad \text { for } x: 0 \text { to } l_{1}, \\
P \frac{\partial^{2} u_{2}}{\partial x^{2}}-\rho_{1} A_{1} \frac{\partial^{2} u_{2}}{\partial t^{2}}=0 \quad \text { for } x: l_{1} \text { to } l_{1}+l_{2}, \\
P \frac{\partial^{2} u_{3}}{\partial x^{2}}-\rho_{1} A_{1} \frac{\partial^{2} u_{3}}{\partial t^{2}}=0 \quad \text { for } x: l_{1}+l_{2} \text { to } l_{1}+l_{2}+l_{3}, \\
E_{2} I_{2} \frac{\partial^{4} v_{1}}{\partial x^{4}}+k_{f} v_{1}+\rho_{2} A_{2} \frac{\partial^{2} v_{1}}{\partial t^{2}}=0 \quad \text { for } x: 0 \text { to } l_{1}, \\
E_{2} I_{2} \frac{\partial^{4} v_{2}}{\partial x^{4}}+k_{f} v_{2}+\rho_{2} A_{2} \frac{\partial^{2} v_{2}}{\partial t^{2}}=0 \quad \text { for } x: l_{1} \text { to } l_{1}+l_{2}, \\
E_{2} I_{2} \frac{\partial^{4} v_{3}}{\partial x^{4}}+k_{f} v_{3}+\rho_{2} A_{2} \frac{\partial^{2} v_{3}}{\partial t^{2}}=0 \quad \text { for } x: l_{1}+l_{2} \text { to } l_{1}+l_{2}+l_{3},
\end{gathered}
$$

where $u_{1}, u_{2}$, and $u_{3}$ are displacements of the fibers; $v_{1}, v_{2}$, and $v_{3}$ are displacements of the substrate; $P$ is string tension; $t$ is time; $k_{f}$ is the constant determined by the material constants of the silicon rubber pad; $A_{1}$ is cross-section area of fibers; $A_{2}$ is cross-section area of substrate; $\rho_{1}$ is density of the fiber material; $\rho_{2}$ is density of the substrate material; $I_{2}$ is the second moment of the cross-sectional area $A_{2} ; E_{2}$ is Young's modulus of the substrate. The optical 
fibers and substrate are bonded at four points. The boundary conditions of the optical fiber coupler are

$$
\begin{aligned}
& u_{1}(0, t)-v_{1}(0, t)=0, \\
& u_{1}\left(l_{1}, t\right)-v_{1}\left(l_{1}, t\right)=0 ， \\
& u_{2}\left(l_{1}, t\right)-v_{2}\left(l_{1}, t\right)=0, \\
& u_{2}\left(l_{1}+l_{2}, t\right)-v_{2}\left(l_{1}+l_{2}, t\right)=0 \text {, } \\
& u_{3}\left(l_{1}+l_{2}, t\right)-v_{3}\left(l_{1}+l_{2}, t\right)=0 \text {, } \\
& u_{3}\left(l_{1}+l_{2}+l_{3}, t\right)-v_{3}\left(l_{1}+l_{2}+l_{3}, t\right)=0 \text {, } \\
& E_{2} I_{2} \frac{\partial^{2} v_{1}(0, t)}{\partial x^{2}}=0, \\
& E_{2} I_{2} \frac{\partial^{3} v_{1}(0, t)}{\partial x^{3}}=0, \\
& v_{1}\left(l_{1}, t\right)-v_{2}\left(l_{1}, t\right)=0, \\
& \frac{\partial v_{1}\left(l_{1}, t\right)}{\partial x}-\frac{\partial v_{2}\left(l_{1}, t\right)}{\partial x}=0, \\
& E_{2} I_{2} \frac{\partial^{2} v_{1}\left(l_{1}, t\right)}{\partial x^{2}}-E_{2} I_{2} \frac{\partial^{2} v_{2}\left(l_{1}, t\right)}{\partial x^{2}}=0, \\
& E_{2} I_{2} \frac{\partial^{3} v_{1}\left(l_{1}, t\right)}{\partial x^{3}}-E_{2} I_{2} \frac{\partial^{3} v_{2}\left(l_{1}, t\right)}{\partial x^{3}}=0, \\
& v_{2}\left(l_{1}+l_{2}, t\right)-v_{3}\left(l_{1}+l_{2}, t\right)=0, \\
& \frac{\partial v_{2}\left(l_{1}+l_{2}, t\right)}{\partial x}-\frac{\partial v_{3}\left(l_{1}+l_{2}, t\right)}{\partial x}=0, \\
& E_{2} I_{2} \frac{\partial^{2} v_{2}\left(l_{1}+l_{2}, t\right)}{\partial x^{2}}-E_{2} I_{2} \frac{\partial^{2} v_{3}\left(l_{1}+l_{2}, t\right)}{\partial x^{2}}=0, \\
& E_{2} I_{2} \frac{\partial^{3} v_{2}\left(l_{1}+l_{2}, t\right)}{\partial x^{3}}-E_{2} I_{2} \frac{\partial^{3} v_{3}\left(l_{1}+l_{2}, t\right)}{\partial x^{3}}=0, \\
& E_{2} I_{2} \frac{\partial^{2} v_{3}\left(l_{1}+l_{2}+l_{3}, t\right)}{\partial x^{2}}=0, \\
& E_{2} I_{2} \frac{\partial^{3} v_{3}\left(l_{1}+l_{2}+l_{3}, t\right)}{\partial x^{3}}=0 .
\end{aligned}
$$

To obtain frequencies, a harmonic movement of the optical fiber coupler is assumed as

$$
\begin{aligned}
& u_{s}(x, t)=\bar{u}_{s}(x) \cos (\omega t) \quad \text { for } s=1,2,3, \\
& v_{s}(x, t)=\bar{v}_{s}(x) \cos (\omega t) \quad \text { for } s=1,2,3
\end{aligned}
$$


where $\bar{u}_{1}(x), \bar{u}_{2}(x), \bar{u}_{3}(x), \bar{v}_{1}(x), \bar{v}_{2}(x)$, and $\bar{v}_{3}(x)$ are vibrational modes, and $\omega$ is the natural frequency of the optical fiber coupler. Substituting (3.3) into (3.1) yields

$$
\begin{gathered}
P \frac{d^{2} \bar{u}_{s}}{d x^{2}}=-\omega^{2} \rho_{1} A_{1} \bar{u}_{s} \quad \text { for } s=1,2,3, \\
E_{2} I_{2} \frac{d^{4} \bar{v}_{s}}{d x^{4}}+k_{f} \bar{v}_{s}=\omega^{2} \rho_{2} A_{2} \bar{v}_{s} \quad \text { for } s=1,2,3 .
\end{gathered}
$$

The boundary conditions of the optical fiber coupler are rewritten as

$$
\begin{aligned}
& \bar{u}_{1}(0)-\bar{v}_{1}(0)=0 \text {, } \\
& \bar{u}_{1}\left(l_{1}\right)-\bar{v}_{1}\left(l_{1}\right)=0 \text {, } \\
& \bar{u}_{2}\left(l_{1}\right)-\bar{v}_{2}\left(l_{1}\right)=0 \text {, } \\
& \bar{u}_{2}\left(l_{1}+l_{2}\right)-\bar{v}_{2}\left(l_{1}+l_{2}\right)=0 \text {, } \\
& \bar{u}_{3}\left(l_{1}+l_{2}\right)-\bar{v}_{3}\left(l_{1}+l_{2}\right)=0, \\
& \bar{u}_{3}\left(l_{1}+l_{2}+l_{3}\right)-\bar{v}_{3}\left(l_{1}+l_{2}+l_{3}\right)=0, \\
& E_{2} I_{2} \frac{d^{2} \bar{v}_{1}(0)}{d x^{2}}=0, \\
& E_{2} I_{2} \frac{d^{3} \bar{v}_{1}(0)}{d x^{3}}=0, \\
& \bar{v}_{1}\left(l_{1}\right)-\bar{v}_{2}\left(l_{1}\right)=0, \\
& \frac{d v_{1}\left(l_{1}\right)}{d x}-\frac{d v_{2}\left(l_{1}\right)}{d x}=0, \\
& E_{2} I_{2} \frac{d^{2} \bar{v}_{1}\left(l_{1}\right)}{d x^{2}}-E_{2} I_{2} \frac{d^{2} \bar{v}_{2}\left(l_{1}\right)}{d x^{2}}=0, \\
& E_{2} I_{2} \frac{d^{3} \bar{v}_{1}\left(l_{1}\right)}{d x^{3}}-E_{2} I_{2} \frac{d^{3} \bar{v}_{2}\left(l_{1}\right)}{d x^{3}}=0, \\
& \bar{v}_{2}\left(l_{1}+l_{2}\right)-\bar{v}_{3}\left(l_{1}+l_{2}\right)=0, \\
& \frac{d \bar{v}_{2}\left(l_{1}+l_{2}\right)}{d x}-\frac{d \bar{v}_{3}\left(l_{1}+l_{2}\right)}{d x}=0, \\
& E_{2} I_{2} \frac{d^{2} \bar{v}_{2}\left(l_{1}+l_{2}\right)}{d x^{2}}-E_{2} I_{2} \frac{d^{2} \bar{v}_{3}\left(l_{1}+l_{2}\right)}{d x^{2}}=0, \\
& E_{2} I_{2} \frac{d^{3} \bar{v}_{2}\left(l_{1}+l_{2}\right)}{d x^{3}}-E_{2} I_{2} \frac{d^{3} \bar{v}_{3}\left(l_{1}+l_{2}\right)}{d x^{3}}=0, \\
& E_{2} I_{2} \frac{d^{2} \bar{v}_{3}\left(l_{1}+l_{2}+l_{3}\right)}{d x^{2}}=0, \\
& E_{2} I_{2} \frac{d^{3} \bar{v}_{3}\left(l_{1}+l_{2}+l_{3}\right)}{d x^{3}}=0 .
\end{aligned}
$$




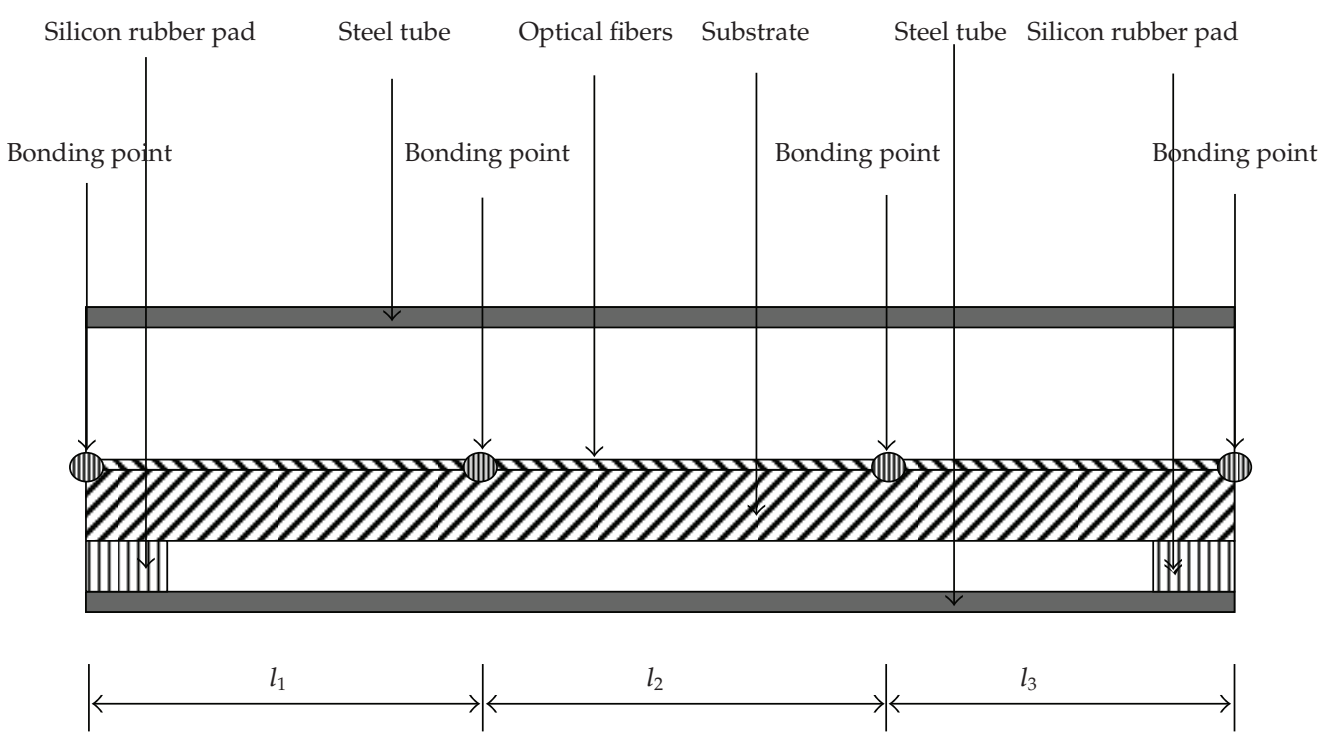

Figure 2: Sectional view of the optical fiber coupler with two spring supports [1, 2].

The equations of motion of the optical fiber coupler can be rearranged in the differential quadrature method formula by substituting (2.1) into (3.4) and (3.5). The equations of motion of the optical fiber coupler are derived as

$$
\begin{gathered}
\sum_{j=1}^{N} \frac{P D_{i, j}^{(2)}}{l_{s}^{2}} \bar{u}_{s, j}=-\omega^{2} \rho_{1} A_{1} \bar{u}_{s, i} \quad \text { for } i=1,2, \ldots, N, s=1,2,3, \\
\sum_{j=1}^{N} \frac{E_{2} I_{2} D_{i, j}^{(4)}}{l_{s}^{4}} \bar{v}_{s, j}+k_{f} \bar{v}_{s, i}=\omega^{2} \rho_{2} A_{2} \bar{v}_{s, i} \quad \text { for } i=1,2, \ldots, N, s=1,2,3 .
\end{gathered}
$$

Using the differential quadrature method, the boundary conditions of the optical fiber coupler can be rearranged into the matrix form as

$$
\begin{gathered}
\bar{u}_{1,1}-\bar{v}_{1,1}=0, \\
\bar{u}_{1, N}-\bar{v}_{1, N}=0, \\
\bar{u}_{2,1}-\bar{v}_{2,1}=0, \\
\bar{u}_{2, N}-\bar{v}_{2, N}=0, \\
\bar{u}_{3,1}-\bar{v}_{3,1}=0, \\
\bar{u}_{3, N}-\bar{v}_{3, N}=0, \\
\sum_{j=1}^{N} \frac{E_{2} I_{2} D_{1, j}^{(2)}}{l_{1}^{2}} \bar{v}_{1, j}=0,
\end{gathered}
$$




$$
\begin{aligned}
& \sum_{j=1}^{N} \frac{E_{2} I_{2} D_{1, j}^{(3)}}{l_{1}^{3}} \bar{v}_{1, j}=0, \\
& \bar{v}_{1, N}-\bar{v}_{2,1}=0, \\
& \sum_{j=1}^{N} \frac{D_{N, j}^{(1)}}{l_{1}} \bar{v}_{1, j}-\sum_{j=1}^{N} \frac{D_{1, j}^{(1)}}{l_{2}} \bar{v}_{2, j}=0, \\
& \sum_{j=1}^{N} \frac{E_{2} I_{2} D_{N, j}^{(2)}}{l_{1}^{2}} \bar{v}_{1, j}-\sum_{j=1}^{N} \frac{E_{2} I_{2} D_{1, j}^{(2)}}{l_{2}^{2}} \bar{v}_{2, j}=0, \\
& \sum_{j=1}^{N} \frac{E_{2} I_{2} D_{N, j}^{(3)}}{l_{1}^{3}} \bar{v}_{1, j}-\sum_{j=1}^{N} \frac{E_{2} I_{2} D_{1, j}^{(3)}}{l_{2}^{3}} \bar{v}_{2, j}=0, \\
& \bar{v}_{2, N}-\bar{v}_{3,1}=0, \\
& \sum_{j=1}^{N} \frac{D_{N, j}^{(1)}}{l_{2}} \bar{v}_{2, j}-\sum_{j=1}^{N} \frac{D_{1, j}^{(1)}}{l_{3}} \bar{v}_{3, j}=0, \\
& \sum_{j=1}^{N} \frac{E_{2} I_{2} D_{N, j}^{(2)}}{l_{2}^{2}} \bar{v}_{2, j}-\sum_{j=1}^{N} \frac{E_{2} I_{2} D_{1, j}^{(2)}}{l_{3}^{2}} \bar{v}_{3, j}=0, \\
& \sum_{j=1}^{N} \frac{E_{2} I_{2} D_{N, j}^{(3)}}{l_{2}^{3}} \bar{v}_{2, j}-\sum_{j=1}^{N} \frac{E_{2} I_{2} D_{1, j}^{(3)}}{l_{3}^{3}} \bar{v}_{3, j}=0, \\
& \sum_{j=1}^{N} \frac{E_{2} I_{2} D_{N, j}^{(2)}}{l_{3}^{2}} \bar{v}_{3, j}=0, \\
& \sum_{j=1}^{N} \frac{E_{2} I_{2} D_{N, j}^{(3)}}{l_{3}^{3}} \bar{v}_{3, j}=0 .
\end{aligned}
$$

\section{The Optical Fiber Coupler with Two Spring Supports}

Figure 2 shows a sectional view of the optical fiber coupler with two rubber pads at each end of the coupler. The rubber pads are placed between the substrate and steel tube. The rubber pads model as the two spring supports. The equations of motion for the optical fiber coupler are $[1,2]$

$$
\begin{gathered}
P \frac{\partial^{2} u_{1}}{\partial x^{2}}-\rho_{1} A_{1} \frac{\partial^{2} u_{1}}{\partial t^{2}}=0 \quad \text { for } x: 0 \text { to } l_{1} \\
P \frac{\partial^{2} u_{2}}{\partial x^{2}}-\rho_{1} A_{1} \frac{\partial^{2} u_{2}}{\partial t^{2}}=0 \quad \text { for } x: l_{1} \text { to } l_{1}+l_{2}
\end{gathered}
$$




$$
\begin{gathered}
P \frac{\partial^{2} u_{3}}{\partial x^{2}}-\rho_{1} A_{1} \frac{\partial^{2} u_{3}}{\partial t^{2}}=0 \quad \text { for } x: l_{1}+l_{2} \text { to } l_{1}+l_{2}+l_{3} \\
E_{2} I_{2} \frac{\partial^{4} v_{1}}{\partial x^{4}}+\rho_{2} A_{2} \frac{\partial^{2} v_{1}}{\partial t^{2}}=0 \quad \text { for } x: 0 \text { to } l_{1} \\
E_{2} I_{2} \frac{\partial^{4} v_{2}}{\partial x^{4}}+\rho_{2} A_{2} \frac{\partial^{2} v_{2}}{\partial t^{2}}=0 \quad \text { for } x: l_{1} \text { to } l_{1}+l_{2} \\
E_{2} I_{2} \frac{\partial^{4} v_{3}}{\partial x^{4}}+\rho_{2} A_{2} \frac{\partial^{2} v_{3}}{\partial t^{2}}=0 \quad \text { for } x: l_{1}+l_{2} \text { to } l_{1}+l_{2}+l_{3}
\end{gathered}
$$

The boundary conditions of the optical fiber coupler are

$$
\begin{aligned}
& u_{1}(0, t)-v_{1}(0, t)=0, \\
& u_{1}\left(l_{1}, t\right)-v_{1}\left(l_{1}, t\right)=0 \text {, } \\
& u_{2}\left(l_{1}, t\right)-v_{2}\left(l_{1}, t\right)=0, \\
& u_{2}\left(l_{1}+l_{2}, t\right)-v_{2}\left(l_{1}+l_{2}, t\right)=0 \text {, } \\
& u_{3}\left(l_{1}+l_{2}, t\right)-v_{3}\left(l_{1}+l_{2}, t\right)=0, \\
& u_{3}\left(l_{1}+l_{2}+l_{3}, t\right)-v_{3}\left(l_{1}+l_{2}+l_{3}, t\right)=0 \text {, } \\
& E_{2} I_{2} \frac{\partial^{2} v_{1}(0, t)}{\partial x^{2}}=0, \\
& E_{2} I_{2} \frac{\partial^{3} v_{1}(0, t)}{\partial x^{3}}=-k_{\text {spring }} v_{1}(0, t), \\
& v_{1}\left(l_{1}, t\right)-v_{2}\left(l_{1}, t\right)=0, \\
& \frac{\partial v_{1}\left(l_{1}, t\right)}{\partial x}-\frac{\partial v_{2}\left(l_{1}, t\right)}{\partial x}=0, \\
& E_{2} I_{2} \frac{\partial^{2} v_{1}\left(l_{1}, t\right)}{\partial x^{2}}-E_{2} I_{2} \frac{\partial^{2} v_{2}\left(l_{1}, t\right)}{\partial x^{2}}=0, \\
& E_{2} I_{2} \frac{\partial^{3} v_{1}\left(l_{1}, t\right)}{\partial x^{3}}-E_{2} I_{2} \frac{\partial^{3} v_{2}\left(l_{1}, t\right)}{\partial x^{3}}=0, \\
& v_{2}\left(l_{1}+l_{2}, t\right)-v_{3}\left(l_{1}+l_{2}, t\right)=0, \\
& \frac{\partial v_{2}\left(l_{1}+l_{2}, t\right)}{\partial x}-\frac{\partial v_{3}\left(l_{1}+l_{2}, t\right)}{\partial x}=0, \\
& E_{2} I_{2} \frac{\partial^{2} v_{2}\left(l_{1}+l_{2}, t\right)}{\partial x^{2}}-E_{2} I_{2} \frac{\partial^{2} v_{3}\left(l_{1}+l_{2}, t\right)}{\partial x^{2}}=0, \\
& E_{2} I_{2} \frac{\partial^{3} v_{2}\left(l_{1}+l_{2}, t\right)}{\partial x^{3}}-E_{2} I_{2} \frac{\partial^{3} v_{3}\left(l_{1}+l_{2}, t\right)}{\partial x^{3}}=0, \\
& E_{2} I_{2} \frac{\partial^{2} v_{3}\left(l_{1}+l_{2}+l_{3}, t\right)}{\partial x^{2}}=0, \\
& E_{2} I_{2} \frac{\partial^{3} v_{3}\left(l_{1}+l_{2}+l_{3}, t\right)}{\partial x^{3}}=k_{\text {spring }} v_{3}\left(l_{1}+l_{2}+l_{3}, t\right) \text {, }
\end{aligned}
$$


where $k_{\text {spring }}$ is the constant determined by material constants of the spring support. Substituting (3.3) into (4) yields

$$
\begin{array}{cc}
P \frac{d^{2} \bar{u}_{s}}{d x^{2}}=-\omega^{2} \rho_{1} A_{1} \bar{u}_{s} \quad \text { for } s=1,2,3 \\
E_{2} I_{2} \frac{d^{4} \bar{v}_{s}}{d x^{4}}=\omega^{2} \rho_{2} A_{2} \bar{v}_{s} \quad \text { for } s=1,2,3 .
\end{array}
$$

The boundary conditions of the optical fiber coupler are rewritten as

$$
\begin{aligned}
& \bar{u}_{1}(0)-\bar{v}_{1}(0)=0 \text {, } \\
& \bar{u}_{1}\left(l_{1}\right)-\bar{v}_{1}\left(l_{1}\right)=0 \text {, } \\
& \bar{u}_{2}\left(l_{1}\right)-\bar{v}_{2}\left(l_{1}\right)=0 \text {, } \\
& \bar{u}_{2}\left(l_{1}+l_{2}\right)-\bar{v}_{2}\left(l_{1}+l_{2}\right)=0 \text {, } \\
& \bar{u}_{3}\left(l_{1}+l_{2}\right)-\bar{v}_{3}\left(l_{1}+l_{2}\right)=0, \\
& \bar{u}_{3}\left(l_{1}+l_{2}+l_{3}\right)-\bar{v}_{3}\left(l_{1}+l_{2}+l_{3}\right)=0 \text {, } \\
& E_{2} I_{2} \frac{d^{2} \bar{v}_{1}(0)}{d x^{2}}=0, \\
& E_{2} I_{2} \frac{d^{3} \bar{v}_{1}(0)}{d x^{3}}=-k_{\text {spring }} \bar{v}_{1}(0), \\
& \bar{v}_{1}\left(l_{1}\right)-\bar{v}_{2}\left(l_{1}\right)=0, \\
& \frac{d v_{1}\left(l_{1}\right)}{d x}-\frac{d v_{2}\left(l_{1}\right)}{d x}=0, \\
& E_{2} I_{2} \frac{d^{2} \bar{v}_{1}\left(l_{1}\right)}{d x^{2}}-E_{2} I_{2} \frac{d^{2} \bar{v}_{2}\left(l_{1}\right)}{d x^{2}}=0, \\
& E_{2} I_{2} \frac{d^{3} \bar{v}_{1}\left(l_{1}\right)}{d x^{3}}-E_{2} I_{2} \frac{d^{3} \bar{v}_{2}\left(l_{1}\right)}{d x^{3}}=0, \\
& \bar{v}_{2}\left(l_{1}+l_{2}\right)-\bar{v}_{3}\left(l_{1}+l_{2}\right)=0, \\
& \frac{d \bar{v}_{2}\left(l_{1}+l_{2}\right)}{d x}-\frac{d \bar{v}_{3}\left(l_{1}+l_{2}\right)}{d x}=0, \\
& E_{2} I_{2} \frac{d^{2} \bar{v}_{2}\left(l_{1}+l_{2}\right)}{d x^{2}}-E_{2} I_{2} \frac{d^{2} \bar{v}_{3}\left(l_{1}+l_{2}\right)}{d x^{2}}=0, \\
& E_{2} I_{2} \frac{d^{3} \bar{v}_{2}\left(l_{1}+l_{2}\right)}{d x^{3}}-E_{2} I_{2} \frac{d^{3} \bar{v}_{3}\left(l_{1}+l_{2}\right)}{d x^{3}}=0, \\
& E_{2} I_{2} \frac{d^{2} \bar{v}_{3}\left(l_{1}+l_{2}+l_{3}\right)}{d x^{2}}=0, \\
& E_{2} I_{2} \frac{d^{3} \bar{v}_{3}\left(l_{1}+l_{2}+l_{3}\right)}{d x^{3}}=k_{\text {spring }} \bar{v}_{3}\left(l_{1}+l_{2}+l_{3}\right) \text {. }
\end{aligned}
$$


The equations of motion of the optical fiber coupler can be rearranged in the differential quadrature method formula by substituting (2.1) into (4.3). The equations of motion of the optical fiber coupler then become

$$
\begin{aligned}
& \sum_{j=1}^{N} \frac{P D_{i, j}^{(2)}}{l_{s}^{2}} \bar{u}_{s, j}=-\omega^{2} \rho_{1} A_{1} \bar{u}_{s, i} \quad \text { for } i=1,2, \ldots, N, s=1,2,3, \\
& \sum_{j=1}^{N} \frac{E_{2} I_{2} D_{i, j}^{(4)}}{l_{s}^{4}} \bar{v}_{s, j}=\omega^{2} \rho_{2} A_{2} \bar{v}_{s, i} \quad \text { for } i=1,2, \ldots, N, s=1,2,3 .
\end{aligned}
$$

Using the differential quadrature method, the boundary conditions of the optical fiber coupler can be rearranged into the matrix form as

$$
\begin{gathered}
\bar{u}_{1,1}-\bar{v}_{1,1}=0, \\
\bar{u}_{1, N}-\bar{v}_{1, N}=0, \\
\bar{u}_{2,1}-\bar{v}_{2,1}=0, \\
\bar{u}_{2, N}-\bar{v}_{2, N}=0, \\
\bar{u}_{3,1}-\bar{v}_{3,1}=0, \\
\bar{u}_{3, N}-\bar{v}_{3, N}=0, \\
\sum_{j=1}^{N} \frac{E_{2} I_{2} D_{1, j}^{(2)} \bar{v}_{1, j}=0,}{l_{1}^{2}} \\
\sum_{j=1}^{N} \frac{E_{2} I_{2} D_{1, j}^{(3)}}{l_{1}^{3}} \bar{v}_{1, j}=-k_{\text {spring }} \bar{v}_{1,1}, \\
\bar{v}_{1, N}-\bar{v}_{2,1}=0, \\
\sum_{j=1}^{N} \frac{D_{N, j}^{(1)}}{l_{1}} \bar{v}_{1, j}-\sum_{j=1}^{N} \frac{D_{1, j}^{(1)}}{l_{2}} \bar{v}_{2, j}=0, \\
\sum_{j=1}^{N} \frac{E_{2} I_{2} D_{N, j}^{(2)}}{l_{1}^{2}} \bar{v}_{1, j}-\sum_{j=1}^{N} \frac{E_{2} I_{2} D_{1, j}^{(2)}}{l_{2}^{2}} \bar{v}_{2, j}=0, \\
\sum_{j=1}^{N} \frac{E_{2} I_{2} D_{N, j}^{(3)}}{l_{1}^{3}} \bar{v}_{1, j}-\sum_{j=1}^{N} \frac{E_{2} I_{2} D_{1, j}^{(3)}}{l_{2}^{3}} \bar{v}_{2, j}=0,
\end{gathered}
$$




$$
\begin{gathered}
\bar{v}_{2, N}-\bar{v}_{3,1}=0, \\
\sum_{j=1}^{N} \frac{D_{N, j}^{(1)}}{l_{2}} \bar{v}_{2, j}-\sum_{j=1}^{N} \frac{D_{1, j}^{(1)}}{l_{3}} \bar{v}_{3, j}=0, \\
\sum_{j=1}^{N} \frac{E_{2} I_{2} D_{N, j}^{(2)}}{l_{2}^{2}} \bar{v}_{2, j}-\sum_{j=1}^{N} \frac{E_{2} I_{2} D_{1, j}^{(2)}}{l_{3}^{2}} \bar{v}_{3, j}=0, \\
\sum_{j=1}^{N} \frac{E_{2} I_{2} D_{N, j}^{(3)}}{l_{2}^{3}} \bar{v}_{2, j}-\sum_{j=1}^{N} \frac{E_{2} I_{2} D_{1, j}^{(3)}}{l_{3}^{3}} \bar{v}_{3, j}=0, \\
\sum_{j=1}^{N} \frac{E_{2} I_{2} D_{N, j}^{(2)}}{l_{3}^{2}} \bar{v}_{3, j}=0, \\
\sum_{j=1}^{N} \frac{E_{2} I_{2} D_{N, j}^{(3)}}{l_{3}^{3}} \bar{v}_{3, j}=k_{\text {spring }} \bar{v}_{3, N} .
\end{gathered}
$$

\section{Nonlinear Dynamic Analysis of an Optical Fiber Coupler with a Continuous Elastic Support}

The nonlinear model assumes that fiber tension varies. The coupler is subjected to a sine shock motion. The equations of motion for the optical fiber coupler with a continuous elastic support are $[1,2]$

$$
\begin{gathered}
P \frac{\partial^{2} u_{1}}{\partial x^{2}}+k_{\text {string }}\left(\int_{0}^{l_{1}} \sqrt{1+\left(\frac{\partial u_{1}}{\partial x}\right)^{2}} d x+\int_{l_{1}}^{l_{1}+l_{2}} \sqrt{1+\left(\frac{\partial u_{2}}{\partial x}\right)^{2}} d x\right. \\
\left.\quad+\int_{l_{1}+l_{2}}^{l_{1}+l_{2}+l_{3}} \sqrt{1+\left(\frac{\partial u_{3}}{\partial x}\right)^{2}} d x-l_{1}-l_{2}-l_{3}\right) \\
-\rho_{1} A_{1} \frac{\partial^{2} u_{1}}{\partial t^{2}}=\rho_{1} A_{1} a \sin (\Omega t) \quad \text { for } x: 0 \text { to } l_{1}, \\
P \frac{\partial^{2} u_{2}}{\partial x^{2}}+k_{\text {string }}\left(\int_{0}^{l_{1}} \sqrt{1+\left(\frac{\partial u_{1}}{\partial x}\right)^{2}} d x+\int_{l_{1}}^{l_{1}+l_{2}} \sqrt{1+\left(\frac{\partial u_{2}}{\partial x}\right)^{2}} d x\right. \\
\left.\quad+\int_{l_{1}+l_{2}}^{l_{1}+l_{2}+l_{3}} \sqrt{1+\left(\frac{\partial u_{3}}{\partial x}\right)^{2}} d x-l_{1}-l_{2}-l_{3}\right) \\
-\rho_{1} A_{1} \frac{\partial^{2} u_{2}}{\partial t^{2}}=\rho_{1} A_{1} a \sin (\Omega t) \quad \text { for } x: l_{1} \text { to } l_{1}+l_{2},
\end{gathered}
$$


Mathematical Problems in Engineering

$$
\begin{gathered}
P \frac{\partial^{2} u_{3}}{\partial x^{2}}+k_{\text {string }}\left(\int_{0}^{l_{1}} \sqrt{1+\left(\frac{\partial u_{1}}{\partial x}\right)^{2}} d x+\int_{l_{1}}^{l_{1}+l_{2}} \sqrt{1+\left(\frac{\partial u_{2}}{\partial x}\right)^{2}} d x\right. \\
\left.+\int_{l_{1}+l_{2}}^{l_{1}+l_{2}+l_{3}} \sqrt{1+\left(\frac{\partial u_{3}}{\partial x}\right)^{2}} d x-l_{1}-l_{2}-l_{3}\right) \\
-\rho_{1} A_{1} \frac{\partial^{2} u_{3}}{\partial t^{2}}=\rho_{1} A_{1} a \sin (\Omega t) \quad \text { for } x: l_{1}+l_{2} \text { to } l_{1}+l_{2}+l_{3}, \\
E_{2} I_{2} \frac{\partial^{4} v_{1}}{\partial x^{4}}+k_{f} v_{1}+\rho_{2} A_{2} \frac{\partial^{2} v_{1}}{\partial t^{2}}=-\rho_{2} A_{2} a \sin (\Omega t) \quad \text { for } x: 0 \text { to } l_{1}, \\
E_{2} I_{2} \frac{\partial^{4} v_{2}}{\partial x^{4}}+k_{f} v_{2}+\rho_{2} A_{2} \frac{\partial^{2} v_{2}}{\partial t^{2}}=-\rho_{2} A_{2} a \sin (\Omega t) \quad \text { for } x: l_{1} \text { to } l_{1}+l_{2}, \\
E_{2} I_{2} \frac{\partial^{4} v_{3}}{\partial x^{4}}+k_{f} v_{3}+\rho_{2} A_{2} \frac{\partial^{2} v_{3}}{\partial t^{2}}=-\rho_{2} A_{2} a \sin (\Omega t) \quad \text { for } x: l_{1}+l_{2} \text { to } l_{1}+l_{2}+l_{3},
\end{gathered}
$$

where $k_{\text {string }}$ is the elastic coefficient of the string, $a$ is the acceleration of shock motion, and $\Omega$ is the circular frequency of shock motion. With the following approximation equation

$$
\sqrt{1+\left(\frac{\partial u_{s}}{\partial x}\right)^{2}} \approx 1+\frac{1}{2}\left(\frac{\partial u_{s}}{\partial x}\right)^{2} \text { for } s=1,2,3
$$

(5.1) can be rewritten as $[1,2]$

$$
\begin{aligned}
& P \frac{\partial^{2} u_{1}}{\partial x^{2}}+\frac{k_{\text {string }}}{2}\left(\int_{0}^{l_{1}}\left(\frac{\partial u_{1}}{\partial x}\right)^{2} d x+\int_{l_{1}}^{l_{1}+l_{2}}\left(\frac{\partial u_{2}}{\partial x}\right)^{2} d x+\int_{l_{1}+l_{2}}^{l_{1}+l_{2}+l_{3}}\left(\frac{\partial u_{3}}{\partial x}\right)^{2} d x\right)-\rho_{1} A_{1} \frac{\partial^{2} u_{1}}{\partial t^{2}} \\
& =\rho_{1} A_{1} a \sin (\Omega t) \quad \text { for } x: 0 \text { to } l_{1} \\
& P \frac{\partial^{2} u_{2}}{\partial x^{2}}+\frac{k_{\text {string }}}{2}\left(\int_{0}^{l_{1}}\left(\frac{\partial u_{1}}{\partial x}\right)^{2} d x+\int_{l_{1}}^{l_{1}+l_{2}}\left(\frac{\partial u_{2}}{\partial x}\right)^{2} d x+\int_{l_{1}+l_{2}}^{l_{1}+l_{2}+l_{3}}\left(\frac{\partial u_{3}}{\partial x}\right)^{2} d x\right)-\rho_{1} A_{1} \frac{\partial^{2} u_{2}}{\partial t^{2}} \\
& =\rho_{1} A_{1} a \sin (\Omega t) \quad \text { for } x: l_{1} \text { to } l_{1}+l_{2}, \\
& P \frac{\partial^{2} u_{3}}{\partial x^{2}}+\frac{k_{\text {string }}}{2}\left(\int_{0}^{l_{1}}\left(\frac{\partial u_{1}}{\partial x}\right)^{2} d x+\int_{l_{1}}^{l_{1}+l_{2}}\left(\frac{\partial u_{2}}{\partial x}\right)^{2} d x+\int_{l_{1}+l_{2}}^{l_{1}+l_{2}+l_{3}}\left(\frac{\partial u_{3}}{\partial x}\right)^{2} d x\right)-\rho_{1} A_{1} \frac{\partial^{2} u_{3}}{\partial t^{2}} \\
& =\rho_{1} A_{1} a \sin (\Omega t) \quad \text { for } x: l_{1}+l_{2} \text { to } l_{1}+l_{2}+l_{3} .
\end{aligned}
$$


The boundary conditions of the optical fiber coupler are

$$
\begin{gathered}
u_{1}(0, t)-v_{1}(0, t)=0, \\
u_{1}\left(l_{1}, t\right)-v_{1}\left(l_{1}, t\right)=0, \\
u_{2}\left(l_{1}, t\right)-v_{2}\left(l_{1}, t\right)=0, \\
u_{2}\left(l_{1}+l_{2}, t\right)-v_{2}\left(l_{1}+l_{2}, t\right)=0, \\
u_{3}\left(l_{1}+l_{2}, t\right)-v_{3}\left(l_{1}+l_{2}, t\right)=0, \\
u_{3}\left(l_{1}+l_{2}+l_{3}, t\right)-v_{3}\left(l_{1}+l_{2}+l_{3}, t\right)=0, \\
E_{2} I_{2} \frac{\partial^{2} v_{1}(0, t)}{\partial x^{2}}=0, \\
E_{2} I_{2} \frac{\partial^{3} v_{1}(0, t)}{\partial x^{3}}=0, \\
E_{2} I_{2} \frac{\partial^{2} v_{2}\left(l_{1}+l_{2}, t\right)}{\partial x^{2}}-E_{2} I_{2} \frac{\partial^{2} v_{3}\left(l_{1}+l_{2}, t\right)-v_{2}\left(l_{1}, t\right)=0}{\partial x^{2}}=0, \\
\frac{\partial^{3} v_{2}\left(l_{1}+l_{2}, t\right)}{\partial x^{3}}-E_{2} I_{2} \frac{\partial^{3} v_{3}\left(l_{1}+l_{2}, t\right)}{\partial x^{3}}=0, \\
\left.E_{2} I_{2} \frac{\partial^{3} v_{3}\left(l_{1}+l_{2}+l_{3}, t\right)}{\partial x^{3}}=0 . l_{2}+l_{3}, t\right) \\
E_{2} I_{2} \frac{\partial^{2} v_{1}\left(l_{1}, t\right)}{\partial x^{2}}-E_{2} I_{2} \frac{\partial^{2} v_{2}\left(l_{1}, t\right)}{\partial x^{2}}=0, \\
E_{2} I_{2} \frac{\partial^{3} v_{1}\left(l_{1}, t\right)}{\partial x^{3}}-E_{2} I_{2} \frac{\partial^{3} v_{2}\left(l_{1}, t\right)}{\partial x^{3}}=0, \\
\left.v_{2}\left(l_{1}+l_{2}, t\right)-v_{3}\left(l_{1}+l_{2}, t\right)=0, t\right) \\
\left.\partial v_{1}, t\right) \\
\frac{\partial v_{3}\left(l_{1}+l_{2}, t\right)}{\partial x}=0,
\end{gathered}
$$

The equation of motion of the optical fiber coupler can be rearranged in the differential quadrature method formula by substituting (2.1) into (5.2) and (5.4). The equations of motion 
of the optical fiber coupler are

$$
\begin{aligned}
& \sum_{j=1}^{N} \frac{P D_{i, j}^{(2)}}{l_{s}^{2}} u_{s, j}+\frac{k_{\text {string }}}{2}\left(\int_{0}^{l_{1}}\left(\frac{\partial u_{1}}{\partial x}\right)^{2} d x+\int_{l_{1}}^{l_{1}+l_{2}}\left(\frac{\partial u_{2}}{\partial x}\right)^{2} d x+\int_{l_{1}+l_{2}}^{l_{1}+l_{2}+l_{3}}\left(\frac{\partial u_{3}}{\partial x}\right)^{2} d x\right)-\rho_{1} A_{1} \frac{\partial^{2} u_{s, i}}{\partial t^{2}} \\
& \quad=\rho_{1} A_{1} a \sin (\Omega t) \quad \text { for } i=1,2, \ldots, N, s=1,2,3, \\
& \sum_{j=1}^{N} \frac{E_{2} I_{2} D_{i, j}^{(4)}}{l_{s}^{4}} v_{s, j}+k_{f} v_{s, i}+\rho_{2} A_{2} \frac{\partial^{2} v_{s, i}}{\partial t^{2}}=-\rho_{2} A_{2} a \sin (\Omega t) \quad \text { for } i=1,2, \ldots, N, s=1,2,3 .
\end{aligned}
$$

Using the differential quadrature method, the boundary conditions of the optical fiber coupler can be rearranged into the matrix form as

$$
\begin{gathered}
u_{1,1}-v_{1,1}=0, \\
u_{1, N}-v_{1, N}=0, \\
u_{2,1}-v_{2,1}=0, \\
u_{2, N}-v_{2, N}=0, \\
u_{3,1}-v_{3,1}=0, \\
u_{3, N}-v_{3, N}=0, \\
\sum_{j=1}^{N} \frac{E_{2} I_{2} D_{1, j}^{(2)}}{l_{1}^{2}} v_{1, j}=0, \\
\sum_{j=1}^{N} \frac{E_{2} I_{2} D_{1, j}^{(3)}}{l_{1}^{3}} v_{1, j}=0, \\
v_{1, N}-v_{2,1}=0, \\
\sum_{j=1}^{N} \frac{D_{N, j}^{(1)}}{l_{1}} v_{1, j}-\sum_{j=1}^{N} \frac{D_{1, j}^{(1)}}{l_{2}} v_{2, j}=0, \\
\sum_{j=1}^{N} \frac{E_{2} I_{2} D_{N, j}^{(2)}}{l_{1}^{2}} v_{1, j}-\sum_{j=1}^{N} \frac{E_{2} I_{2} D_{1, j}^{(2)}}{l_{2}^{2}} v_{2, j}=0, \\
\sum_{j=1}^{N} \frac{E_{2} I_{2} D_{N, j}^{(3)}}{l_{1}^{3}} v_{1, j}-\sum_{j=1}^{N} \frac{E_{2} I_{2} D_{1, j}^{(3)}}{l_{2}^{3}} v_{2, j}=0, \\
v_{2, N}-v_{3,1}=0,
\end{gathered}
$$




$$
\begin{gathered}
\sum_{j=1}^{N} \frac{D_{N, j}^{(1)}}{l_{2}} v_{2, j}-\sum_{j=1}^{N} \frac{D_{1, j}^{(1)}}{l_{3}} v_{3, j}=0, \\
\sum_{j=1}^{N} \frac{E_{2} I_{2} D_{N, j}^{(2)}}{l_{2}^{2}} v_{2, j}-\sum_{j=1}^{N} \frac{E_{2} I_{2} D_{1, j}^{(2)}}{l_{3}^{2}} \bar{v}_{3, j}=0, \\
\sum_{j=1}^{N} \frac{E_{2} I_{2} D_{N, j}^{(3)}}{l_{2}^{3}} v_{2, j}-\sum_{j=1}^{N} \frac{E_{2} I_{2} D_{1, j}^{(3)}}{l_{3}^{3}} v_{3, j}=0, \\
\sum_{j=1}^{N} \frac{E_{2} I_{2} D_{N, j}^{(2)}}{l_{3}^{2}} v_{3, j}=0, \\
\sum_{j=1}^{N} \frac{E_{2} I_{2} D_{N, j}^{(3)}}{l_{3}^{3}} v_{3, j}=0 .
\end{gathered}
$$

\section{Numerical Results and Discussion}

Figure 3 presents the natural frequencies of the optical fiber coupler for various values of $P$. The material and geometric parameters of the optical fiber coupler are $k_{f}=50000 \mathrm{~N} / \mathrm{m}^{2}$, $A_{1}=3.1 \times 10^{-8} \mathrm{~m}^{2}, A_{2}=6.61 \times 10^{-6} \mathrm{~m}^{2}, \rho_{1}=2.2 \times 10^{3} \mathrm{~kg} / \mathrm{m}^{3}, \rho_{2}=2.2 \times 10^{3} \mathrm{~kg} / \mathrm{m}^{3}, I_{2}=$ $4.34 \times 10^{-12} \mathrm{~m}^{4}, l_{1}=0.1333 \mathrm{~m}, l_{2}=0.1333 \mathrm{~m}, l_{3}=0.1333 \mathrm{~m}$, and $E_{2}=7.24 \times 10^{10} \mathrm{~N} / \mathrm{m}^{2}[1,2]$. The first and second natural frequencies of the optical fiber coupler are robust to the string tension, $P$. The third and fourth natural frequencies of the optical fiber coupler increase as the string tension, $P$, increases. Figure 4 shows the natural frequencies of the optical fiber coupler for various values of $k_{f}$. The material and geometric parameters of the optical fiber coupler are $P=0.01 \mathrm{~N}, A_{1}=3.1 \times 10^{-8} \mathrm{~m}^{2}, A_{2}=6.61 \times 10^{-6} \mathrm{~m}^{2}, \rho_{1}=2.2 \times 10^{3} \mathrm{~kg} / \mathrm{m}^{3}, \rho_{2}=2.2 \times 10^{3} \mathrm{~kg} / \mathrm{m}^{3}$, $I_{2}=4.34 \times 10^{-12} \mathrm{~m}^{4}, l_{1}=0.1333 \mathrm{~m}, l_{2}=0.1333 \mathrm{~m}, l_{3}=0.1333 \mathrm{~m}$, and $E_{2}=7.24 \times 10^{10} \mathrm{~N} / \mathrm{m}^{2}$ $[1,2]$. The first and third natural frequencies of the optical fiber coupler increase as the rubber pad stiffness increases. The rubber pad stiffness does not significantly affect the second and fourth natural frequencies of the optical fiber coupler. Figure 5 lists the natural frequencies of the optical fiber coupler with bonding points at various locations. The material and geometric parameters of the optical fiber coupler are $P=0.01 \mathrm{~N}, k_{f}=50000 \mathrm{~N} / \mathrm{m}^{2}, A_{1}=3.1 \times 10^{-8} \mathrm{~m}^{2}$, $A_{2}=6.61 \times 10^{-6} \mathrm{~m}^{2}, \rho_{1}=2.2 \times 10^{3} \mathrm{~kg} / \mathrm{m}^{3}, \rho_{2}=2.2 \times 10^{3} \mathrm{~kg} / \mathrm{m}^{3}, I_{2}=4.34 \times 10^{-12} \mathrm{~m}^{4}, l_{1}+l_{2}+l_{3}=$ $0.4 \mathrm{~m}$, and $E_{2}=7.24 \times 10^{10} \mathrm{~N} / \mathrm{m}^{2}[1,2]$. The fourth natural frequency of the optical fiber coupler generally increases rapidly as lengths $l_{1}$ and $l_{3}$ increase. The locations of bonding points markedly impact the second and third natural frequencies of the optical fiber coupler. Figure 6 plots the natural frequencies of the optical fiber coupler for various values of $k_{\text {spring. }}$. The material and geometric parameters of the optical fiber coupler are $P=0.01 \mathrm{~N}, A_{1}=$ $3.1 \times 10^{-8} \mathrm{~m}^{2}, A_{2}=6.61 \times 10^{-6} \mathrm{~m}^{2}, \rho_{1}=2.2 \times 10^{3} \mathrm{~kg} / \mathrm{m}^{3}, \rho_{2}=2.2 \times 10^{3} \mathrm{~kg} / \mathrm{m}^{3}, I_{2}=4.34 \times 10^{-12} \mathrm{~m}^{4}$, $l_{1}=0.1333 \mathrm{~m}, l_{2}=0.1333 \mathrm{~m}, l_{3}=0.1333 \mathrm{~m}$, and $E_{2}=7.24 \times 10^{10} \mathrm{~N} / \mathrm{m}^{2}[1,2]$. The spring

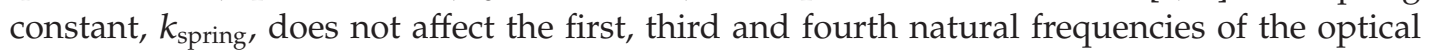
fiber coupler. Notably, the spring constant, $k_{\text {spring }}$, increases the second natural frequencies of the optical fiber coupler. Figures 7 and 8 show the displacements of the center of the fibers 


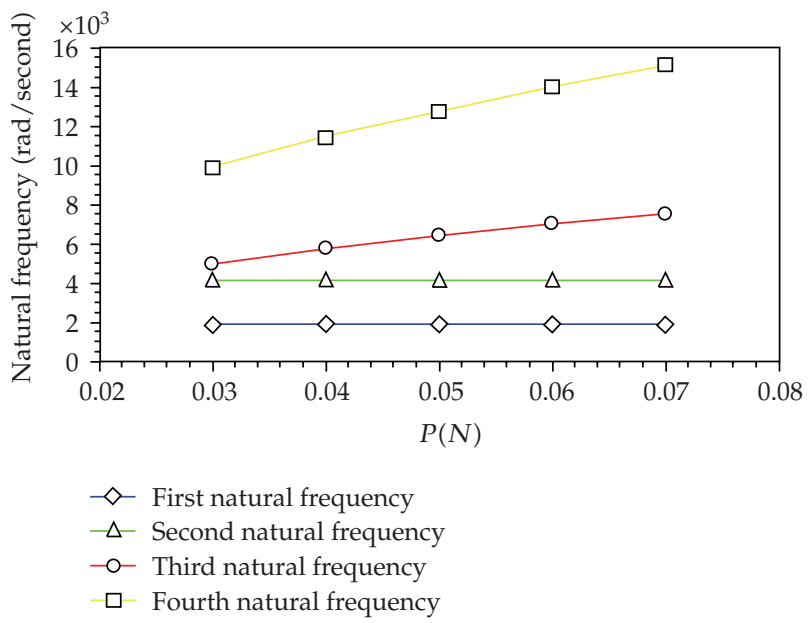

Figure 3: Natural frequencies of the optical fiber coupler for various values of $P$.

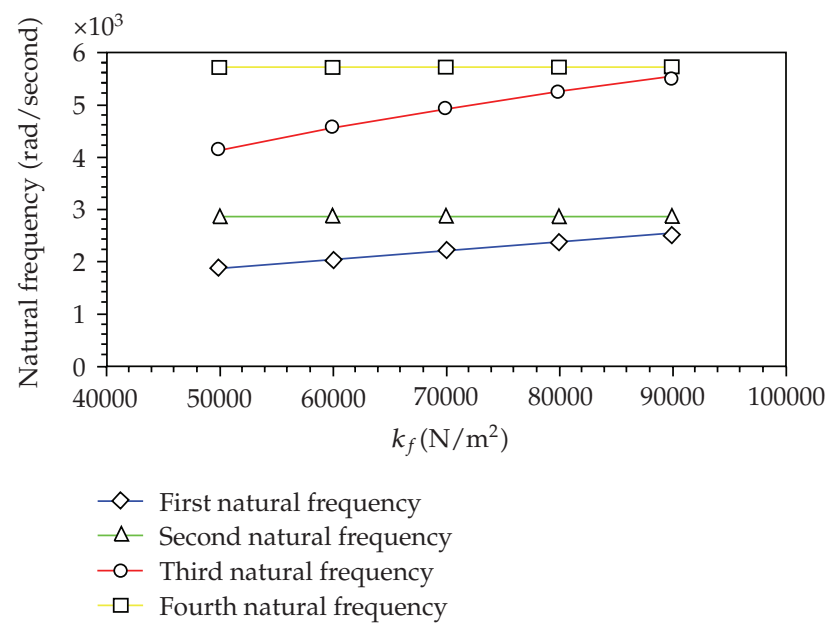

Figure 4: Natural frequencies of the optical fiber coupler for various values of $k_{f}$.

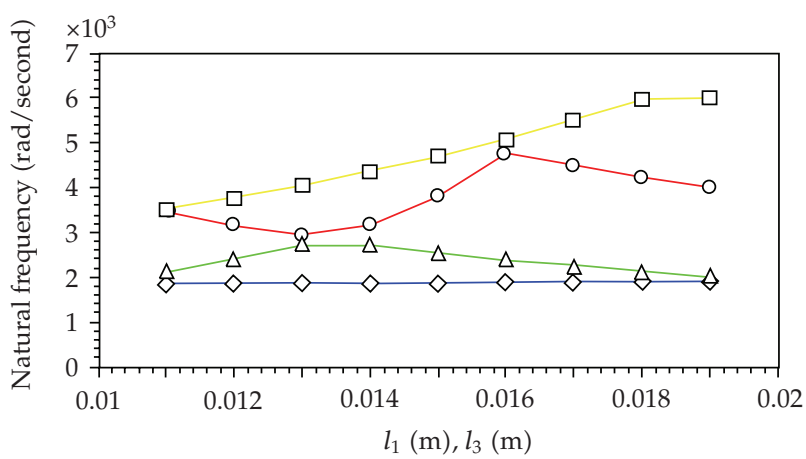

$\checkmark-$ First natural frequency
$\Delta-$ Second natural frequency
- - Third natural frequency
$\square-$ Fourth natural frequency

Figure 5: Natural frequencies of the optical fiber coupler with bonding points at various locations. 


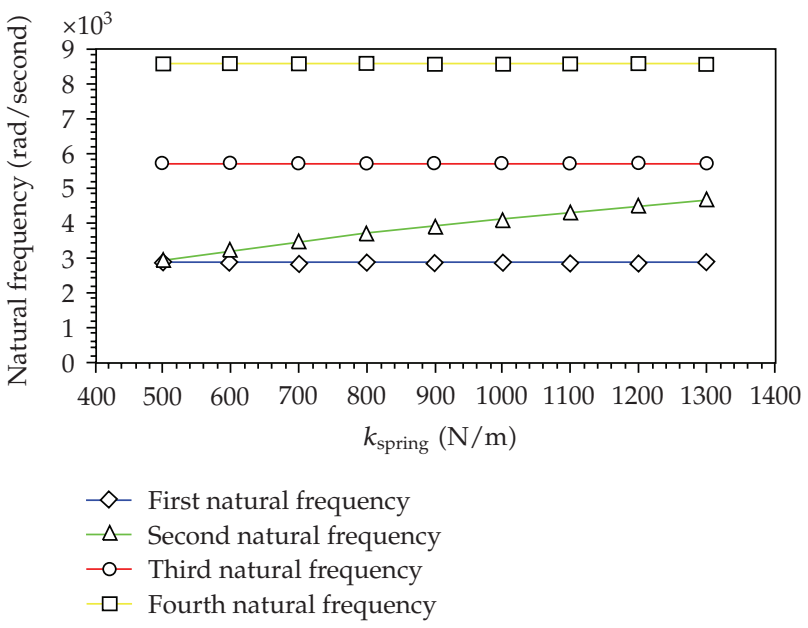

Figure 6: Natural frequencies of the optical fiber coupler for various values of $k_{\text {spring }}$.

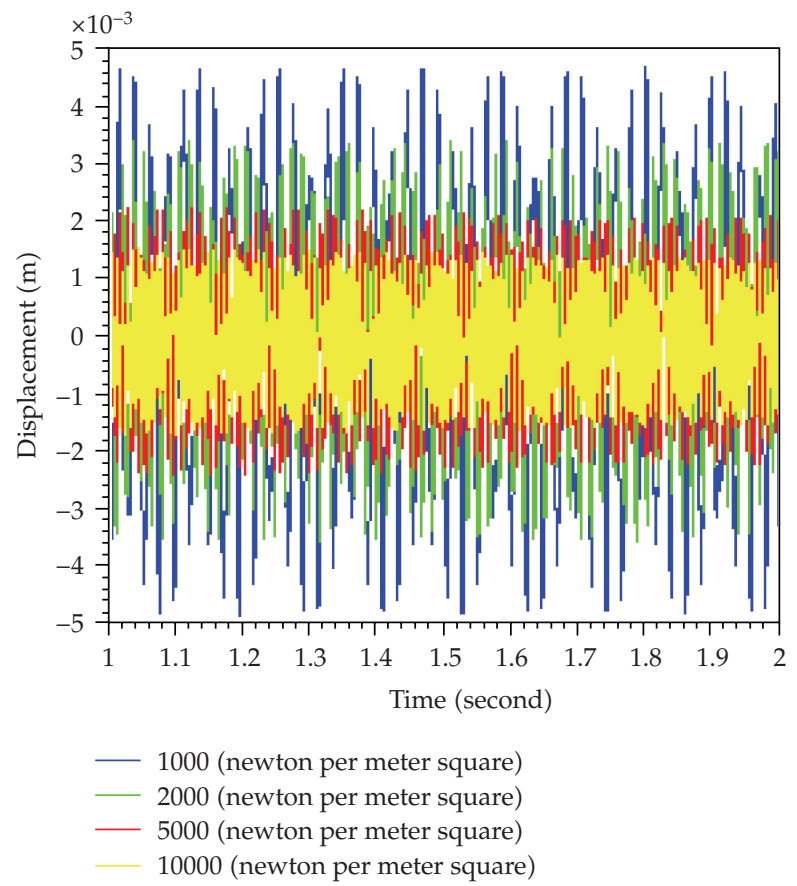

Figure 7: Displacements at the fiber center for various values of $k_{f}$.

and the substrate under a shock, respectively. The material and geometric parameters of the optical fiber coupler are $P=0.01 \mathrm{~N}, A_{1}=3.1 \times 10^{-8} \mathrm{~m}^{2}, A_{2}=6.61 \times 10^{-6} \mathrm{~m}^{2}, \rho_{1}=2.2 \times 10^{3} \mathrm{~kg} / \mathrm{m}^{3}$, $\rho_{2}=2.2 \times 10^{3} \mathrm{~kg} / \mathrm{m}^{3}, I_{2}=4.34 \times 10^{-12} \mathrm{~m}^{4}, l_{1}=0.1333 \mathrm{~m}, l_{2}=0.1333 \mathrm{~m}, l_{3}=0.1333 \mathrm{~m}, E_{2}=$ $7.24 \times 10^{10} \mathrm{~N} / \mathrm{m}^{2}$, and $k_{\text {string }}=5000 \mathrm{~N} / \mathrm{m}[1,2]$. The fibers and substrate stiffen when the foundation stiffness, $k_{f}$, is large. The differential quadrature method is effective in treating this problem. 


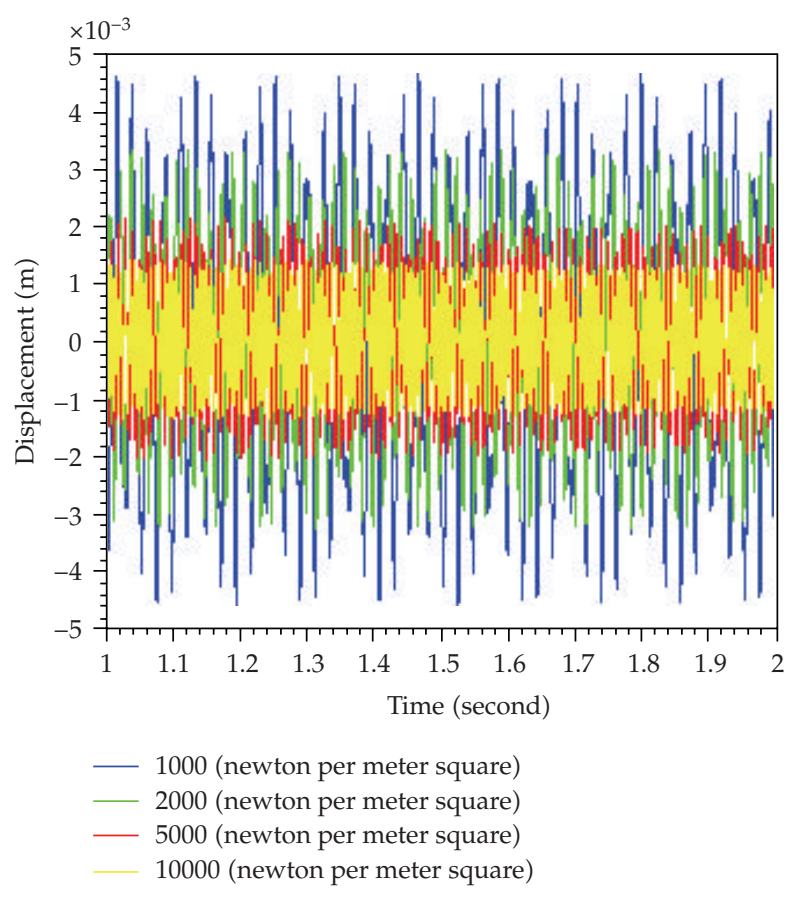

Figure 8: Displacements at the substrate center for various values of $k_{f}$.

\section{Conclusions}

This study demonstrates the value of the differential quadrature method for vibration analysis of an optical fiber coupler. The effects of string tension $P$, bonding locations, surrounding medium, spring constant $k_{\text {spring, }}$ and rubber pad stiffness $k_{f}$ on the natural frequencies of the optical fiber coupler are discussed. The effect of stiffness of the silicon rubber pad during vibrations is significant and should be incorporated into the designs of the optical fiber couplers.

\section{References}

[1] G. Cheng and J. W. Zu, "Dynamic analysis of an optical fiber coupler in telecommunications," Journal of Sound and Vibration, vol. 268, no. 1, pp. 15-31, 2003.

[2] C. Sun, Vibration analysis of an optical fiber coupler, M.S. thesis, University of Toronto, Toronto, Canada, 2001.

[3] B. A. Malomed and R. S. Tasgal, "Vibration modes of a gap soliton in a nonlinear optical medium," Physical Review E, vol. 49, no. 6, pp. 5787-5796, 1994.

[4] G. D. Brown, S. E. Spence, and J. P. Ingold, "Vibration testing of fiber optic compoents," in Fiber Optics Reliability: Benign and Adverse Environments III, vol. 1174 of Proceedings of the SPIE, pp. 167-170, 1990.

[5] L.-S. Huang, S.-S. Lee, E. Motamedi, M. C. Wu, and C.-J. Kim, "Optical coupling analysis and vibration characterization for packaging of $2 \times 2$ MEMS vertical torsion mirror switches," in Microelectronic Structures and MEMS for Optical Processing IV, vol. 3513 of Proceedings of SPIE, pp. 135-143, 1998.

[6] W. J. Thomes Jr., F. V. LaRocca, R. C. Switzer, M. N. Ott, R. F. Chuska, and S. L. Macmurphy, “Vibration performance comparison study on current fiber optic connector technologies," in Optical Technologies for Arming, Safing, Fuzing, and Firing IV, vol. 7070 of Proceedings of SPIE, pp. 70700A.1-70700A.15, 2008. 
[7] K. M. Liew, J.-B. Han, Z. M. Xiao, and H. Du, “Differential quadrature method for Mindlin plates on Winkler foundations," International Journal of Mechanical Sciences, vol. 38, no. 4, pp. 405-421, 1996.

[8] K. M. Liew, J.-B. Han, and Z. M. Xiao, "Differential quadrature method for thick symmetric cross-ply laminates with first-order shear flexibility," International Journal of Solids and Structures, vol. 33, no. 18, pp. 2647-2658, 1996.

[9] J.-B. Han and K. M. Liew, "An eight-node curvilinear differential quadrature formulation for Reissner/Mindlin plates," Computer Methods in Applied Mechanics and Engineering, vol. 141, no. 3-4, pp. 265-280, 1997.

[10] K. M. Liew and F.-L. Liu, "Differential cubature method: a solution technique for Kirchhoff plates of arbitrary shape," Computer Methods in Applied Mechanics and Engineering, vol. 145, no. 1-2, pp. 1-10, 1997.

[11] K. M. Liew and T. M. Teo, "Modeling via differential quadrature method: three-dimensional solutions for rectangular plates," Computer Methods in Applied Mechanics and Engineering, vol. 159, no. 3-4, pp. 369-381, 1998.

[12] F.-L. Liu and K. M. Liew, "Differential quadrature element method: a new approach for free vibration analysis of polar Mindlin plates having discontinuities," Computer Methods in Applied Mechanics and Engineering, vol. 179, no. 3-4, pp. 407-423, 1999.

[13] K. M. Liew and J.-B. Han, "A four-node differential quadrature method for straight-sided quadrilateral Reissner/Mindlin plates," Communications in Numerical Methods in Engineering, vol. 13, no. 2, pp. 73-81, 1999.

[14] K. M. Liew, T. M. Teo, and J.-B. Han, "Comparative accuracy of DQ and HDQ methods for threedimensional vibration analysis of rectangular plates," International Journal for Numerical Methods in Engineering, vol. 45, no. 12, pp. 1831-1848, 1999.

[15] H. Du, K. M. Liew, and M. K. Lim, "Generalized differential quadrature method for buckling analysis," Journal of Engineering Mechanics, vol. 122, no. 2, pp. 95-100, 1996.

[16] J.-B. Han and K. M. Liew, "Axisymmetric free vibration of thick annular plates," International Journal of Mechanical Sciences, vol. 41, no. 9, pp. 1089-1109, 1999.

[17] A. N. Sherbourne and M. D. Pandey, "Differential quadrature method in the buckling analysis of beams and composite plates," Computers and Structures, vol. 40, no. 4, pp. 903-913, 1991.

[18] P. Mirfakhraei and D. Redekop, "Buckling of circular cylindrical shells by the differential quadrature method," International Journal of Pressure Vessels and Piping, vol. 75, no. 4, pp. 347-353, 1998.

[19] S. Tomasiello, "Differential quadrature method: application to initial-boundary-value problems," Journal of Sound and Vibration, vol. 218, no. 4, pp. 573-585, 1998.

[20] S. Moradi and F. Taheri, "Delamination buckling analysis of general laminated composite beams by differential quadrature method," Composites Part B, vol. 30, no. 5, pp. 503-511, 1999.

[21] M. A. De Rosa and C. Franciosi, "Exact and approximate dynamic analysis of circular arches using DQM," International Journal of Solids and Structures, vol. 37, no. 8, pp. 1103-1117, 2000.

[22] J.-A. Sun and Z.-Y. Zhu, "Upwind local differential quadrature method for solving incompressible viscous flow," Computer Methods in Applied Mechanics and Engineering, vol. 188, no. 1-3, pp. 495-504, 2000.

[23] M. Tanaka and W. Chen, "Dual reciprocity BEM applied to transient elastodynamic problems with differential quadrature method in time," Computer Methods in Applied Mechanics and Engineering, vol. 190, no. 18-19, pp. 2331-2347, 2001.

[24] W. Chen and T. Zhong, "The study on the nonlinear computations of the DQ and DC methods," Numerical Methods for Partial Differential Equations, vol. 13, no. 1, pp. 57-75, 1997.

[25] F. Civan, "Solving multivariable mathematical models by the quadrature and cubature methods," Numerical Methods for Partial Differential Equations, vol. 10, no. 5, pp. 545-567, 1994.

[26] L. Hua and K. Y. Lam, "Frequency characteristics of a thin rotating cylindrical shell using the generalized differential quadrature method," International Journal of Mechanical Sciences, vol. 40, no. 5, pp. 443-459, 1998.

[27] X. Wang and H. Gu, "Static analysis of frame structures by the differential quadrature element method," International Journal for Numerical Methods in Engineering, vol. 40, no. 4, pp. 759-772, 1997.

[28] X. Wang, J. Yang, and J. Xiao, “On free vibration analysis of circular annular plates with non-uniform thickness by the differential quadrature method," Journal of Sound and Vibration, vol. 184, no. 3, pp. 547-551, 1995.

[29] X. Wang, M. Tan, and Y. Zhou, "Buckling analyses of anisotropic plates and isotropic skew plates by the new version differential quadrature method," Thin-Walled Structures, vol. 41, no. 1, pp. 15-29, 2003. 
[30] X. Wang and C. W. Bert, "A new approach in applying differential quadrature to static and free vibrational analyses of beams and plates," Journal of Sound and Vibration, vol. 162, no. 3, pp. 566-572, 1993.

[31] C. W. Bert and M. Malik, "Differential quadrature method in computational mechanics: a review," Applied Mechanics Reviews, vol. 49, no. 1, pp. 1-28, 1996. 


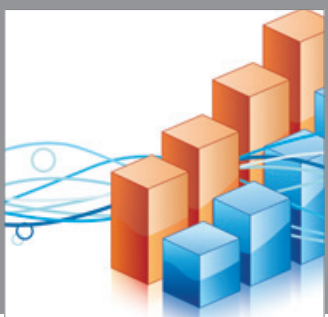

Advances in

Operations Research

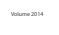

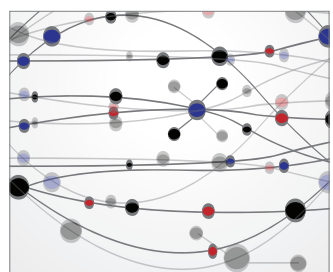

\section{The Scientific} World Journal
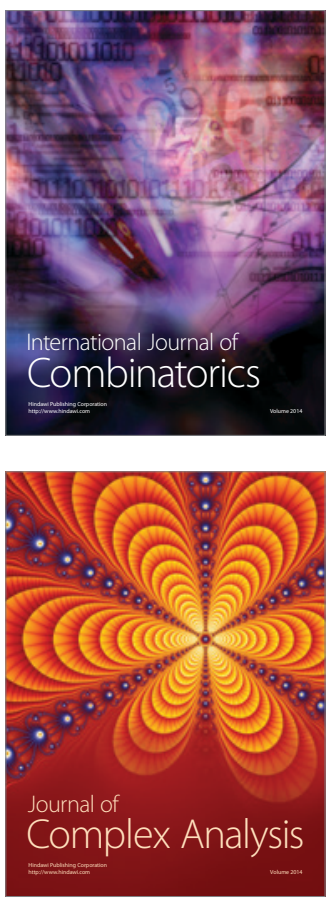

International Journal of

Mathematics and

Mathematical

Sciences
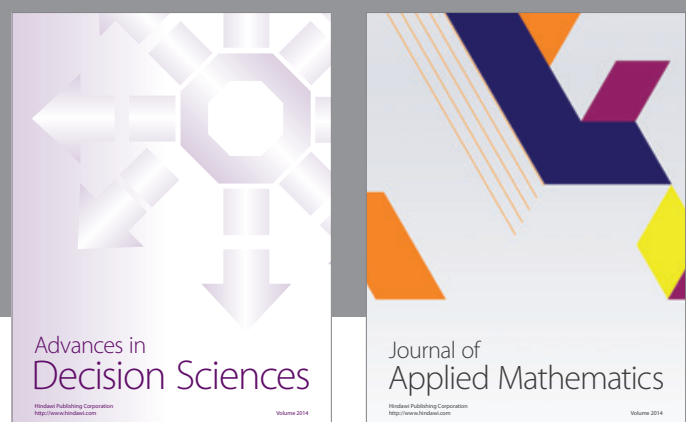

Journal of

Applied Mathematics
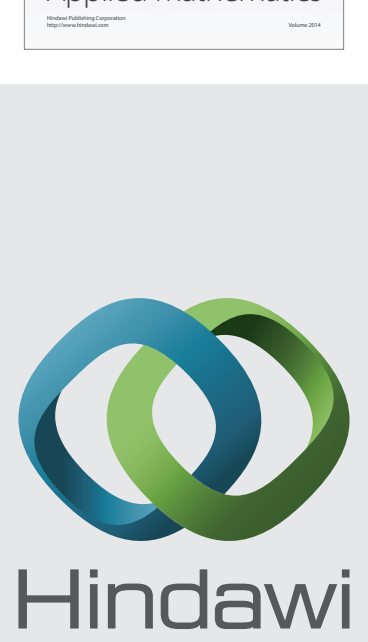

Submit your manuscripts at http://www.hindawi.com
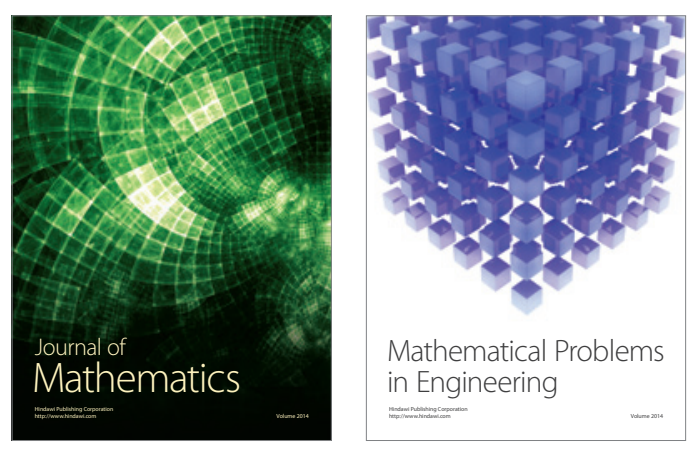

Mathematical Problems in Engineering
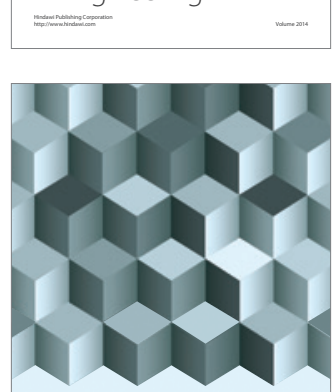

Journal of

Function Spaces
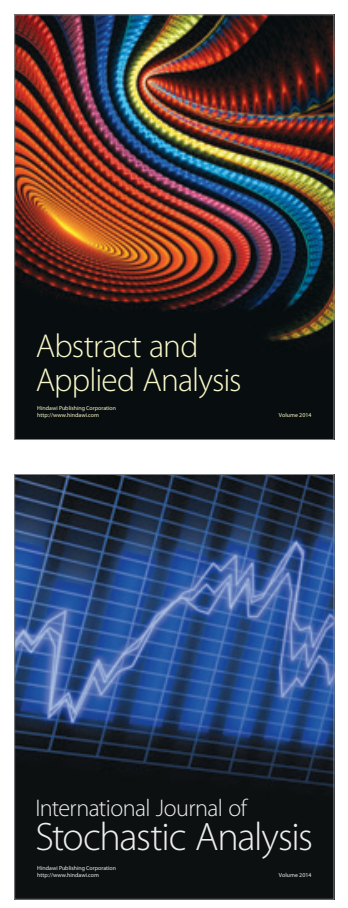

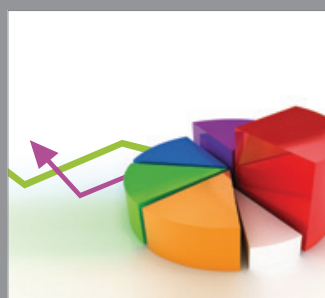

ournal of

Probability and Statistics

Promensencen
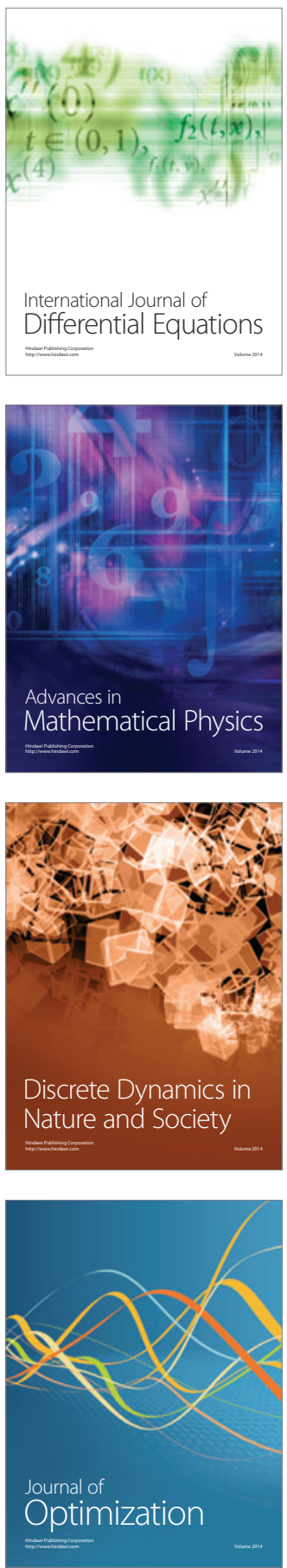